\title{
Nefropatia e Retinopatia em Diabéticos do Tipo 1 de um Programa de Atendimento Multiprofissional Universitário
}

\section{artigo original}

\section{EMERSON SAMPAIO \\ HenRiQueta G.G. Almeida Vinicius Daher Alvares Delfino}

Ambulatório Multiprofissional de Diabetes, Hospital das Clínicas, Universidade Estadual de Londrina, PR.

Recebido em 06/04/06 Revisado em 26/07/06 Aceito em 16/10/06

\section{RESUMO}

O diabetes mellitus (DM) tipo 1 é associado a complicações crônicas micro e macrovasculares. Propusemo-nos a estudar a associação entre nefropatia (ND) e retinopatia diabéticas (RD), e destas com variáveis clínico-laboratoriais em portadores de DM tipo 1 acompanhados em um centro universitário. Realizou-se um estudo transversal através de análise de prontuários. Foram estudados 81 pacientes; a prevalência de RD foi $21,0 \%$ e de ND, 35,8\%; houve associação entre ambas. Os portadores de ND e RD possuíam maior tempo de DM, maior prevalência de hipertensão, pior controle glicêmico e LDL colesterol mais elevado. A taxa de filtração glomerular (TFG) foi associada ao tempo de DM, hipertensão e albuminúria. Identificou-se menor TFG nos portadores de complicações microvasculares, principalmente naqueles com lesões avançadas. Concluímos que, em nosso estudo com portadores de DM tipo 1, houve associação entre RD, ND e TFG, sendo tempo de DM e hipertensão variáveis associadas de forma independente. (Arq Bras Endocrinol Metab 2007;51/3:410-418)

Descritores: Diabetes mellitus tipo 1; Retinopatia diabética; Nefropatia diabética; Taxa de filtração glomerular

\section{ABSTRACT}

Nephropathy and Retinopathy in Type 1 Diabetics Assisted by a Universitary Multiprofessional Program.

Type 1 diabetes mellitus (DM) is linked to micro and macrovascular longterm complications. We proposed to study the association between diabetic nephropathy (DN) and retinopathy (DR), and of these complications with clinical-laboratory variables in type $1 \mathrm{DM}$ patients followed in a universitary center. It was a transversal study through medical registry analysis. We studied 81 patients; prevalence of DR was $21.0 \%$ and of DN was $35.8 \%$, there was association between the complications. Carriers of DN and DR have greater DM duration, greater hypertension prevalence, worse glycemic control and greater LDL cholesterol. It was found reduced glomerular filtration rate (GFR) in patients with microvascular complications, mainly in the ones with advanced lesions. In our study there was association between DR, ND and GFR, DM duration and hypertension were independently associated variables. (Arq Bras Endocrinol Metab 2007;51/3:410-418)

Keywords: Type 1 diabetes mellitus; Diabetic retinopathy; Diabetic nephropathy; Glomerular filtration rate 
$\mathrm{O}$ DIABETES MELLITUS (DM) tipo 1 é associado a complicações crônicas micro e macrovasculares, as quais comprometem estruturas de órgãos como rins, olhos, nervos, vasos e coração (1). A retinopatia diabética (RD) e a nefropatia diabética (ND) são duas complicações temidas; elas contribuem para elevação da morbidade e mortalidade, impondo enormes custos médicos, econômicos e sociais para o paciente e para o sistema público de saúde (2). Embora os processos fisiopatológicos destas lesões não estejam completamente elucidados, a hiperglicemia representa um papel fundamental nesse fenômeno (3).

A RD é a complicação ocular mais severa (4) e está entre as principais causas de cegueira irreversível no Brasil e no mundo (5). Os sinais clínicos iniciais da sua presença incluem a presença de microaneurismas, pequenos exsudatos (extravasamento de lipoproteínas) e hemorragias puntiformes intrarretinianas, que estão presentes em quase todos os portadores de DM tipo 1 após cerca de 20 anos de doença (6). Apesar do controle glicêmico poder diminuir a progressão da retinopatia já estabelecida, parte dos portadores de DM irá, a despeito do controle glicêmico, desenvolver ou avançar a doença ocular, sugerindo que outros fatores estão envolvidos nesse processo $(7,8)$.

O DM é a causa mais importante de doença renal terminal em países industrializados (9). Embora esse grupo de pacientes seja representado principalmente por diabéticos do tipo 2, em alguns países como a Finlândia, a ND em portadores de DM tipo 1 responde por dois terços dos pacientes com doença renal terminal (10). O desenvolvimento de microalbuminúria persistente é um marcador estabelecido para a ND (11-13). Um terço dos portadores de DM tipo 1 podem desenvolver microalbuminúria e cerca de $15 \%$ a $20 \%$ desenvolvem macroalbuminúria em 20 anos do diagnóstico do DM $(14,15)$, informação relevante não só do ponto de vista renal, pois há uma associação entre proteinúria e doença cardiovascular, uma importante causa de mortalidade neste grupo $(16,17)$.

Propusemo-nos, portanto, estudar a associação entre ND e RD, assim como a associação entre a ocorrência destas complicações e as seguintes variáveis clínico-laboratoriais: sexo, idade, tempo de doença, índice de massa corpórea (IMC), hipertensão, controle glicêmico, colesterol sérico total, LDL colesterol, HDL colesterol, trigliceridemia e estimativa da taxa de filtração glomerular (TFG) em diabéticos do tipo 1 acompanhados em um centro universitário de referência.

\section{PACIENTES E MÉTODOS}

Foram estudadas informações obtidas através de prontuários médicos de 81 pacientes portadores de DM tipo 1 acompanhados pelo Ambulatório de Atendimento Multiprofissional de Diabetes, Disciplina de Endocrinologia da Universidade Estadual de Londrina, de forma transversal. Foram incluídos todos os pacientes portadores de DM tipo 1 com mais de 5 anos de diagnóstico e desenvolvimento sexual adulto (segundo os critérios de Tanner) atendidos no período de 2003 e 2004. Os dados foram coletados após a última visita realizada neste período.

Os pacientes foram acompanhados através de avaliação clínica por equipe multiprofissional (médicos, enfermeiras, nutricionista, psicóloga e assistente social) e por avaliação bioquímica. DM tipo 1 foi definido como idade de surgimento antes dos 40 anos e insulinodependência no primeiro ano após o diagnóstico; nos casos de pacientes obesos, critérios clínicos, ocorrência de cetoacidose diabética e presença de acantose nigricans, e laboratoriais, dosagem de peptídeo C basal e auto-anticorpos (anti-GAD, anti-IA2 e anti-insulina), foram avaliados concomitantemente. O IMC foi definido como a razão do peso sobre a altura ao quadrado, e classificado de acordo com os critérios do National Institutes of Health (1998) (18). Hipertensão foi definida até os 18 anos como pressão arterial sistólica e/ou diastólica $\geq 95^{\circ}$ percentil para gênero, idade e altura, e nos adultos como sistólica $>130 \mathrm{mmHg}$ e/ou diastólica $>80 \mathrm{mmHg}$, medida por método auscultatório, ou uso regular de anti-hipertensivos, conforme critérios da American Diabetes Association (ADA) (19). As avaliações laboratoriais foram realizadas no laboratório do Hospital Universitário de Londrina. A determinação da creatinina sérica foi realizada através de método químico não enzimático com leitura colorimétrica; colesterol total, colesterol HDL e trigliceridemia por métodos enzimáticos específicos com leitura colorimétrica (todas essas análises utilizaram kits produzidos por Dade Behring, Estados Unidos). Os níveis de LDL colesterol foram estimados através da equação de Friedewald (20). Avaliamos todas as determinações de hemoglobina glicada total (somatório das frações Ala, Alb e Alc) realizadas no período de um ano da consulta estudada (a análise laboratorial da hemoglobina glicada foi realizada através de cromatografia com resina de troca iônica, kit produzido por Alamar Tecno Científica Ltda., Brasil).

A avaliação oftalmológica foi realizada através de oftalmoscopia indireta sob midríase e, se necessário, retinografia com fluoresceína. O diagnóstico foi categorizado em ausência de $\mathrm{RD}, \mathrm{RD}$ não proliferativa e $\mathrm{RD}$ proliferativa; a classificação das alterações retinianas utilizou critérios do Early Treatment Diabetic Retinopathy Study (1991) (21). As avaliações oftalmológicas estiveram a cargo da Disciplina de Oftalmologia da mesma instituição.

A pesquisa da ND foi realizada através de microalbuminúria noturna de 12 horas por ensaio turbidimétrico (kit produzido por APTEC Diagnostics, Bélgica) ou proteinúria de 24 horas por método químico com leitura co- 
lorimétrica nos portadores de macroalbuminúria (kit produzido por Dade Behring, Estados Unidos). Foram considerados pacientes portadores de albuminúria positiva aqueles com pelo menos duas ou mais coletas positivas, num período de seis meses pregressos ao estudo. Foram considerados microalbuminúricos os pacientes com excreção urinária de albumina entre 20 e $199 \mu \mathrm{g} / \mathrm{min}$ e macroalbuminúricos os com excreção maior ou igual a $200 \mu \mathrm{g} / \mathrm{min}$, conforme os critérios da ADA (19). Também foram considerados portadores de ND os pacientes em terapia dialítica substitutiva ( 2 pacientes) ou receptores de transplante renal (1 paciente) em decorrência de ND diagnosticada previamente. A TFG foi estimada através da equação de Schwartz (22) em pacientes até 18 anos de idade, e pela de Cockcroft-Gault nos acima de 18 anos (23).

Para avaliar diferenças entre as médias das variáveis contínuas, após estratificação, foi utilizado o teste $t$ de Student ou a Análise de Variância, quando as pressuposições dos testes eram satisfeitas. Para avaliar as pressuposições foram utilizados o teste Kolmogorov-Smirnov para testar normalidade e o de Barlett para avaliar a homogeneidade das variâncias entre os diversos grupos. Quando as pressuposições não foram preenchidas, optou-se por testes não paramétricos: Mann-Whitney e Kruskal-Wallis. Utilizou-se o teste qui-quadrado para avaliar associação entre variáveis discretas. As variáveis também foram estudadas através de estudo de correlação, sendo usado o coeficiente de correlação de Pearson e o teste da sua significância. Foram selecionadas para análise de regressão multivariada as variáveis com $\mathrm{p}<0,1$ na análise univariada. As variáveis contínuas foram expressas como média \pm desvio-padrão (DP). Em todos os testes estatísticos foi utilizado o nível de significância -de $5 \%(\mathrm{p}<0,05)$.

\section{RESULTADOS}

\section{Descrição da população}

Dos 81 pacientes estudados, $52(64,2 \%)$ eram do sexo feminino. A média de idade foi de $26,4 \pm 8,7$ anos, variando de 15 a 56 anos, sendo que $72(88,9 \%)$ possuíam mais de 18 anos de idade. A média do tempo de doença desde o diagnóstico inicial de DM foi de $13,4 \pm 5,8$ anos, com um mínimo de 5,3 anos e um máximo de 30,8 anos. Quanto ao IMC, uma paciente $(1,2 \%)$ foi portadora de baixo peso (IMC $<18), 62$ $(76,5 \%)$ tinham peso normal, $15(18,5 \%)$ sobrepeso, 1 $(1,2 \%)$ obesidade classe I, I ( $1,2 \%)$ obesidade classe II e $1(1,2 \%)$ obesidade classe III. Em relação à pressão arterial sistêmica, $60(74,1 \%)$ eram normotensos e 21 $(25,9 \%)$ eram hipertensos.

Quanto às avaliações oftalmológicas, 64 $(79,0 \%)$ foram normais, $7(8,6 \%)$ revelaram retinopatia não proliferativa e $10(12,3 \%)$, retinopatia proliferativa. Em relação à pesquisa urinária, identificamos que 52 pacientes $(64,2 \%)$ possuíam albuminúria negativa, $17(21,0 \%)$ microalbuminúria e $12(14,8 \%)$ macroalbuminúria. A TFG média foi de $124,8 \pm 48,6 \mathrm{ml} /$ $\mathrm{min} / 1,73 \mathrm{~m}^{2}$, variando de zero a $260,6 \mathrm{ml} /$ $\mathrm{min} / 1,73 \mathrm{~m}^{2}$. Três pacientes $(3,7 \%)$ realizavam terapia dialítica substitutiva (diálise peritoneal) e uma paciente $(1,2 \%)$ havia sido submetida a transplante renal por ND.

A média da hemoglobina glicada total no último ano foi de $10,1 \pm 1,8 \%$, do colesterol total de $190,3 \pm 39,6 \mathrm{mg} / \mathrm{dl}$, LDL colesterol de 119,2 $\pm 33,3$ $\mathrm{mg} / \mathrm{dl}, \mathrm{HDL}$ colesterol de 48,8 $\pm 12,3 \mathrm{mg} / \mathrm{dl} \mathrm{e}$ trigliceridemia de $113,5 \pm 80,1 \mathrm{mg} / \mathrm{dl}$. Apesar de 60 pacientes $(74,1 \%)$ possuírem LDL colesterol maior ou igual a $100 \mathrm{mg} / \mathrm{dl}$, apenas 8 pacientes $(9,8 \%)$ faziam uso de estatina.

\section{Análise das variáveis associadas à RD}

Não foram identificadas associações significativas entre RD e as variáveis: sexo, IMC, níveis séricos de colesterol total, fração HDL colesterol ou trigliceridemia. Identificamos que os portares de $\mathrm{RD}$ eram mais velhos, com maior tempo de DM e apresentavam maior prevalência de hipertensão. A análise de regressão multivariada evidenciou que a idade $(\mathrm{p}=0,78)$ é uma possível variável de confusão, quando avaliada concomitantemente à variável tempo de $\mathrm{DM}(\mathrm{p}=0,01)$. Laboratorialmente, evidenciamos pior controle glicêmico no último ano, LDL colesterol mais elevado e menor estimativa da TFG (tabela 1). Os portadores de $\mathrm{RD}$ proliferativa possuíam maior tempo de doença que os de não proliferativa (figura 1 ).

\section{Análise das variáveis associadas à ND e TFG}

Não foram identificadas associações significativas entre ND e as variáveis: idade, IMC, níveis séricos de colesterol total, fração HDL colesterol ou trigliceridemia. Identificamos que os portadores de ND possuíam maior tempo de DM, assim como maior prevalência do sexo feminino e hipertensão. Laboratorialmente, evidenciamos pior controle glicêmico no último ano, LDL colesterol mais elevado e menor estimativa da TFG (tabela 2). Os portadores de macroalbuminúria possuíam maior tempo de doença que os de microalbuminúria (figura 2).

A análise multivariada evidenciou que tempo de $\mathrm{DM}(\mathrm{p}<0,01)$, presença de albuminúria $(\mathrm{p}=0,03)$ e hipertensão $(p=0,01)$ foram variáveis associadas com a TFG de forma independente. A TFG estimada também foi maior entre os portadores de microalbuminúria em relação aos portadores de macroalbuminúria, sendo de $131,5 \pm 26,4 \mathrm{ml} / \mathrm{min} / 1,73 \mathrm{~m}^{2}$ e 66,5 $\pm 39,7 \mathrm{ml} / \mathrm{min} / 1,73 \mathrm{~m}^{2}$, respectivamente $(\mathrm{p}<0,01)$, 
Tabela 1. Variáveis clínico-laboratoriais e retinopatia diabética (RD).

\begin{tabular}{lccc}
\hline Variáveis & Sem RD & Com RD & p \\
Sexo feminino & $41(78,8 \%)$ & $11(21,2 \%)$ & $0,96^{*}$ \\
Sexo masculino & $23(79,3 \%)$ & $6(20,7 \%)$ & $0,96^{*}$ \\
Idade (anos) & $25,0 \pm 8,1$ & $31,6 \pm 9,5$ & $0,00^{\dagger}$ \\
Tempo de doença (anos) & $12,1 \pm 5,5$ & $18,4 \pm 3,8$ & $0,00^{\dagger}$ \\
IMC (kg/m²) & $23,3 \pm 3,2$ & $24,1 \pm 5,4$ & $0,53^{\ddagger}$ \\
Hipertensos & $7(33,3 \%)$ & $14(66,7 \%)$ & $0,00^{*}$ \\
Normotensos & $57(95,0 \%)$ & $3(5,0 \%)$ & $0,00^{*}$ \\
Hb A1 total $(\%)$ & $9,9 \pm 1,7$ & $10,8 \pm 2,0$ & $0,0^{\dagger}$ \\
Coleterol total (mg/dl) & $186,5 \pm 40,9$ & $204,5 \pm 31,4$ & $0,1^{\dagger}$ \\
LDL colesterol (mg/dl) & $115,2 \pm 33,5$ & $134,5 \pm 28,3$ & $0,0^{\dagger}$ \\
HDL colesterol (mg/dl) & $48,6 \pm 11,1$ & $49,4 \pm 16,3$ & $0,88^{\ddagger}$ \\
Triglicérides (mg/dl) & $111,1 \pm 81,3$ & $122,5 \pm 76,9$ & $0,60^{\dagger}$ \\
TFG ml/min/1,73m² & $137,9 \pm 40,3$ & $85,7 \pm 37,4$ & $0,0^{\dagger}$ \\
\hline
\end{tabular}

* Teste qui-quadrado; ${ }^{\dagger}$ Teste $t$ de Student; ${ }^{\ddagger}$ Teste Mann-Whitney.

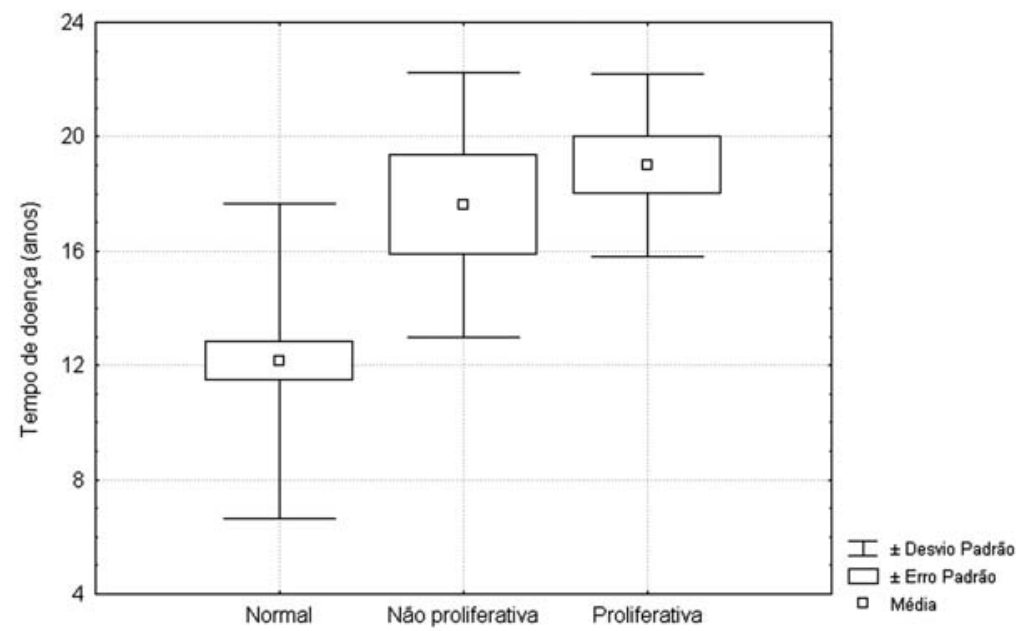

Figura 1. Tempo médio de diagnóstico de DM e formas de retinotopia diabética (Análise de Variância entre os três grupos: $p<0,01$ )

Tabela 2. Variáveis clínico-laboratoriais e nefropatia diabética (ND).

\begin{tabular}{lccc}
\hline Variáveis & Sem ND & Com ND & p \\
Sexo feminino & $29(55,8 \%)$ & $23(44,2 \%)$ & $0,03^{*}$ \\
Sexo masculino & $23(79,3 \%)$ & $6(20,7 \%)$ & $0,0^{*}$ \\
Idade (anos) & $25,7 \pm 9,4$ & $27,6 \pm 7,4$ & $0,36^{\dagger}$ \\
Tempo de doença (anos) & $12,4 \pm 5,9$ & $15,4 \pm 5,0$ & $0,2^{\dagger}$ \\
IMC (kg/m²) & $23,6 \pm 2,9$ & $23,4 \pm 4,9$ & $0,29^{\ddagger}$ \\
Hipertensos & $8(38,1 \%)$ & $13(61,9 \%)$ & $0,00^{*}$ \\
Normotensos & $44(73,3 \%)$ & $16(26,7 \%)$ & $0,00^{*}$ \\
Hb A1 total (\%) & $9,8 \pm 1,6$ & $10,6 \pm 2,0$ & $0,04^{\dagger}$ \\
Coleterol total (mg/dl) & $185,5 \pm 42,5$ & $198,9 \pm 32,8$ & $0,14^{\dagger}$ \\
LDL colesterol (mg/dl) & $113,0 \pm 34,6$ & $130,4 \pm 28,1$ & $0,02^{\dagger}$ \\
HDL colesterol (mg/dl) & $48,0 \pm 11,6$ & $50,2 \pm 13,6$ & $0,43{ }^{\dagger}$ \\
Triglicérides (mg/dl) & $120,3 \pm 87,8$ & $101,2 \pm 63,4$ & $0,31^{\dagger}$ \\
TFG ml/min/1,73m² & $139,4 \pm 36,3$ & $104,6 \pm 50,6$ & $0,00^{\ddagger}$ \\
\hline
\end{tabular}

* Teste qui-quadrado; ${ }^{\dagger}$ Teste $t$ de Student; ${ }^{\ddagger}$ Teste Mann-Whitney. 


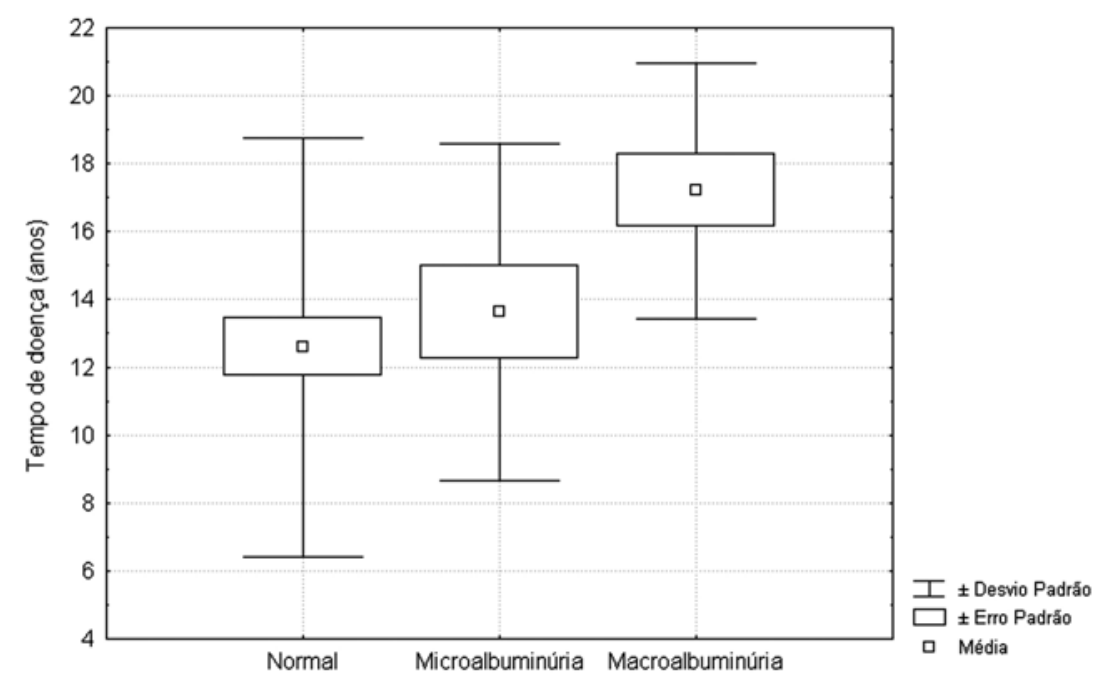

Figura 2. Tempo médio de diagnóstico de DM e graus de albumínia (Análise de Variância entre três grupos: $p<0,01$ )

assim como foi maior entre os normotensos que entre os hipertensos, sendo suas TFGs $137,5 \pm 41,4$ $\mathrm{ml} / \mathrm{min} / 1,73 \mathrm{~m}^{2}$ e $88,6 \pm 50,3 \mathrm{ml} / \mathrm{min} / 1,73 \mathrm{~m}^{2}$, respectivamente $(\mathrm{p}<0,01)$. Houve correlação negativa entre o tempo de doença e a taxa de filtração glomerular $(\mathrm{r}=-0,46 ; \mathrm{p}<0,01)$.

\section{Associaçāo RD e ND}

Houve associação entre a ocorrência de RD e ND ( $<<$ $0,01)$. Entre os portadores de retinopatia, $88,2 \%$ eram portadores de albuminúria, enquanto que, entre os portadores de nefropatia, 51,7\% tinham comprometimento retiniano. Houve uma razão de prevalência da RD e ND significativa de 1,75 (IC 95\%: 1,36-2,91). É importante salientar que, apesar da existência desta associação, em 19,8\% dos casos não houve concordância quanto às complicações, sendo que em 17,3\% dos pacientes ocorreu ND isolada e em 2,5\% dos casos, $\mathrm{RD}$ isolada (tabela 3 ).

Houve uma associação significativa entre a presença de RD e macroalbuminúria. Identificamos que a prevalência de macroalbuminúria foi de 58,8\% nos portadores de RD e de apenas $3,1 \%$ nos pacientes sem lesão retiniana $(\mathrm{p}<0,01)$. Também houve associação significativa entre a presença de ND e RD proliferativa. A prevalência da RD proliferativa foi de 31,0\% nos portadores de ND e de apenas 2,0\% nos pacientes normoalbuminúricos $(\mathrm{p}<0,01)$.

A análise de regressão multivariada evidenciou que, dentre as variáveis avaliadas, hipertensão e tempo de doença foram associadas com ND e RD de forma independente, as demais variáveis avaliadas (idade, sexo, Hb Al total, LDL colesterol) não foram significativas (tabela 4 ).

\section{DISCUSSĀO}

A duração do $\operatorname{DM}(15,24)$, a hipertensão $(14,24)$ e o controle glicêmico $(8,25)$ já foram apontados como fatores de risco em estudos prospectivos tanto para ND como para RD. A associação identificada em nosso estudo entre as complicações e as variáveis hipertensão e controle glicêmico podem decorrer da perpetuação de fatores de risco há muito tempo estabelecidos, contribuindo para progressão da lesão renal e retiniana. Destaca-se que apenas tempo de diagnóstico de DM e hipertensão foram variáveis independentes em nossa análise.

Nos pacientes portadores de DM tipo 1 , o tempo de evolução do DM é o fator mais importante e de reconhecida relação com a prevalência e incidência da $\mathrm{RD}$ (6). Em relação ao acometimento renal, a duração do DM também constitui um fator de risco. $\mathrm{O}$ conceito que ND raramente surge após 30 anos de doença vem sendo combatido; estudo prospectivo realizado por Arun e cols. (2003) evidenciou surgimento e progressão da ND em pacientes portadores de DM tipo 1 com mais de 30 anos de evolução de DM (16). Durante a evolução da 
Tabela 3. Concordância entre retinopatia e nefropatia diabéticas nos pacientes estudados.

\begin{tabular}{lccc}
\hline Retinopatia & Normoalbuminúria & Microalbuminúria & Macroalbuminúria \\
Ausente & $50(61,7 \%)$ & $12(14,8 \%)$ & $2(2,5 \%)$ \\
Não-proliferativa & $1(1,2 \%)$ & $3(3,7 \%)$ & $3(3,7 \%)$ \\
Proliferativa & $1(1,2 \%)$ & $2(2,5 \%)$ & $7(8,6 \%)$ \\
\hline
\end{tabular}

Qui-quadrado entre os diagnósticos de oftalmoscopia e albuminúria: p<0,01.

Tabela 4. Análise de regressão multivariada das variáveis associadas à retinopatia e à nefropatia diabéticas.

\begin{tabular}{lcccc}
\hline Variáveis estudadas & \multicolumn{2}{c}{ Retinopatia diabética } & \multicolumn{2}{c}{ Nefropatia diabética } \\
& - & $\mathbf{p}$ & - & $\mathbf{p}$ \\
Idade $^{*}$ & 0,00 & 0,78 & - & - \\
Sexo $^{\dagger}$ & - & - & 0,21 & 0,06 \\
Hipertensão $_{\text {Tempo de doença }}$ & $0,51 \neq$ & $0,00 \neq$ & $0,29 \neq$ & $0,02 \ddagger$ \\
Hb A1 total & $0,02 \ddagger$ & $0,01 \neq$ & $0,01 \neq$ & $0,00 \ddagger$ \\
LDL colesterol & 0,01 & 0,53 & 0,02 & 0,53 \\
\hline
\end{tabular}

* Variável incluída apenas na análise da retinopatia diabética.

† Variável incluída apenas na análise da nefropatia diabética.

‡ Coeficientes estatisticamente significativos.

ND, há surgimento ou intensificação da hipertensão. No entanto, esta não é apenas conseqüência da presença da ND, pois os níveis pressóricos elevados e a predisposição à hipertensão são importantes determinantes da susceptibilidade à ND. A história familial de hipertensão é encontrada mais freqüentemente em pais de pacientes com DM tipo l e ND do que nos genitores de diabéticos com albuminúria normal (26). Aumento significativo da pressão arterial tem sido reportado em pacientes com DM tipo 1 com discreto aumento na excreção urinária de albumina, mas sem alteração na TFG, situação em que a hipertensão não pode ser facilmente atribuída à doença renal (27). A hipertensão também está envolvida no desenvolvimento e na progressão da RD $(28,29)$, uma vez que em portadores de DM há perda da regulação da perfusão retiniana aumentando a susceptibilidade à lesão decorrente da hipertensão arterial por aumento do fluxo sangüíneo e lesão de células endoteliais na retina (30).

A ausência de significância, na análise multivariada, para o nível de controle glicêmico possivelmente foi decorrente de baixa precisão dos ensaios, pois a instituição não dispunha de ensaio específico para a fração da $\mathrm{HbAlc}$, e a forma de processamento das dosagens para hemoglobina $\mathrm{Al}$ utilizada não foi automatizada, o que desencadeou uma variabilidade analítica importante.
A dislipidemia vem sendo apontada como fator de risco para o desenvolvimento das complicações microvasculares em portadores de DM tipo 1, sendo sua associação descrita por diversos autores $(31,32)$. Há uma interação entre a lipemia e a progressão da $\mathrm{ND}$, independentemente do controle pressórico e glicêmico. A natureza desse fenômeno é diferente em estágios distintos da doença renal; enquanto a progressão dos pacientes com normoalbuminúria para albuminúria patológica é associada a níveis elevados de LDL colesterol, nos pacientes já portadores de microalbuminúria a progressão é associada a níveis de trigliceridemia e, nos portadores de macroalbuminúria, ao tamanho das partículas de LDL colesterol (32). O mecanismo pelo qual as partículas lipídicas poderiam participar no processo da gênese da RD não é claro; modelo experimental aponta a oxidação de LDL colesterol mediada por hiperglicemia como uma forma de promoção de estresse oxidativo, uma potencial via para lesão retiniana em portadores de DM (33).

Dahlquist e cols. (2001) acompanharam uma coorte de portadores de DM tipo l por uma média de 29 anos, evidenciando as variações da TFG neste período. A TFG se caracteriza por apresentar uma elevação no período inicial, após diagnóstico do DM. A hiperfiltração glomerular inicial é um preditor fraco, mas significativo para a ND. Durante o decorrer da 
doença, há uma queda mais acelerada da TFG nos portadores de macroalbuminúria em relação aos portadores de microalbuminúria e desses em relação aos normoalbuminúricos, dados concordantes com as menores TFG encontradas nos portadores de ND em nosso estudo (15). Enquanto a hiperfiltração glomerular possui um poder de predição fraco, as taxas de albuminúria e os níveis pressóricos são os principais fatores de risco para evolução da ND (34).

A associação entre $\mathrm{RD}$ e $\mathrm{ND}$ em portadores de DM tipo 1 é descrita na literatura (2). Estudos prospectivos evidenciaram que, quando realiza-se o diagnóstico de lesão retiniana, há um risco maior para surgimento da ND (35), assim como também há um risco maior para $\mathrm{RD}$ em pacientes portadores de microalbuminúria (24). No entanto, um subgrupo de pacientes apresenta lesão diagnosticada apenas na retina ou no rim. Parte desse fenômeno é explicada pelo método diagnóstico utilizado. Estudo realizado por Chavers e cols. (1994), utilizando para avaliação de ND a dosagem de microalbuminúria (funcional) e técnicas de análise morfométricas em glomérulos oriundos de biopsias renais em diabéticos do tipo 1 (morfológicas) e pesquisando RD através de lesões estruturais retinianas, encontraram discordância marcante em um grupo de pacientes que se apresentavam como normoalbuminúricos, sem lesão estrutural glomerular, mas com avançada retinopatia (36). Klein e cols. (2005) também realizaram o estudo da associação $\mathrm{RD}$ e lesões glomerulares pré-clínicas; a avaliação renal incluiu índices mesângio-glomerulares obtidos através de análise por microscopia eletrônica, metodologia essa que identificou presença de alterações glomerulares microscópicas relacionadas à ND em cerca de 50\% dos pacientes sem sinais de RD (37). Em nosso estudo, identificou-se maior prevalência de ND que RD, sendo que em 17,3\% dos casos ocorreu o diagnóstico de ND sem diagnóstico concomitante de $\mathrm{RD}$; no entanto, há autores que encontraram dados discordantes aos que descrevemos utilizando o mesmo método de investigação (38). A elevada razão de prevalência entre ND e RD tem repercussão na prática clínica, há necessidade de pesquisar diagnósticos diferenciais para causa de proteinúria, mesmo em portadores de DM tipo 1 , nos casos de aparecimento súbito de macroalbuminúria sem lesões retinianas concomitantes (39). No entanto, a alta prevalência de portadores de ND isolada encontrada neste estudo pode decorrer do subdiagnóstico da RD, pois enquanto o diagnóstico de ND foi realizado a partir de um critério laboratorial (albuminúria), o diagnóstico de RD foi realizado através de oftalmoscopia indireta pelo serviço de oftalmologia em um hospital escola universitário.
A correlação entre gênero e ND não é unanimidade na literatura. Em nosso estudo, encontramos associação entre o sexo feminino e ND, enquanto estudos prospectivos não apontaram associação entre ambos (35) ou até mesmo sugeriram que o sexo masculino fosse fator de risco (14). Em nossa análise, informações sobre alguns outros fatores de risco, como o tabagismo e raça, não foram avaliadas. Pela natureza retrospectiva do estudo, optamos por não coletar informações não-padronizadas no prontuário médico do serviço. Devido a tratar-se de um estudo transversal, não foi possível estudar a influência dos níveis pressóricos sobre as complicações microvasculares.

O combate aos fatores de risco é de suma importância antes da instalação das complicações, pois são esses, associados à predisposição genética, que desencadeiam a seqüência fisiopatológica que culmina com a ocorrência das lesões de órgãos-alvo nos pacientes diabéticos. A intensificação do tratamento metabólico dos portadores de DM tipo 1 está envolvida na redução da incidência da ND e doença renal terminal evidenciada nas últimas quatro décadas (17). O diagnóstico precoce das complicações tem importância clínica, pois há evidências de que a progressão das lesões da ND pode ser retardada através de diversas estratégias terapêuticas, que incluem um controle glicêmico estrito $(8,25)$, controle da hipertensão $(40,41)$ e bloqueio do sistema renina-angiotensina (42). Houve avanço no tratamento da RD com a fotocoagulação com argônio $(43,44)$ e a vitrectomia $(45)$, e também há evidências de que a evolução da RD pode ser retardada no portador de DM tipo 1 pelo controle glicêmico estrito $(8,25)$ e que o controle pressórico é essencial durante a terapêutica. As evidências do benefício do tratamento da hipertensão sobre a RD derivam principalmente de estudos realizados em portadores de DM tipo 2 (46). Há indícios de que o bloqueio do sistema renina-angiotensina também possa contribuir no combate à RD, apesar de serem necessários maiores estudos nessa área (47). Uma vez que há associação entre RD e ND, a indicação de bloqueio do sistema renina-angiotensina para portadores de $\mathrm{RD}$ hipertensos é benéfica, pois apesar dos questionamentos em relação à eficácia específica sobre a $\mathrm{RD}$, o benefício sobre a evolução renal e a redução da mortalidade cardiovascular desta intervenção nestes pacientes são largamente comprovados e justificam a indicação (48).

Em conclusão, nos portadores de DM tipo 1 em acompanhamento ambulatorial no serviço estudado, houve associação entre ND e RD. Ambas as complicações crônicas estiveram associadas às variáveis tempo de DM e hipertensão. A TFG esteve associada 
de forma independente ao tempo de doença, hipertensão e albuminúria. Identificou-se menores TFGs nos portadores de complicações crônicas microvasculares, sendo a redução ainda maior nos estágios mais avançados dessas complicações.

\section{REFERÊNCIAS}

1. Nathan DM. Long-term complications of diabetes mellitus. N Engl J Med 1993;328:1676-85.

2. Jawa A, Kcomt J, Fonseca VA. Diabetic nephropathy and retinopathy. Med Clin N Am 2004;88:1001-36.

3. Brownlee M. Biochemistry and molecular cell biology of diabetic complications. Nature 2001;414:813-20.

4. Frank RN. Diabetic retinopathy. N Engl J Med 2004;350:4858.

5. Vilela MP, Assdi AK, Pletsch L, Giacomet A. Inquérito entre pacientes e médicos sobre as estratégias aplicadas na prevenção e tratamento da retinopatia diabética. Arq Bras Oftalmol 1997;60:152-5.

6. Klein R, Klein BEK, Moss SE, Davis MD, DeMets DL. The Wisconsin epidemiologic study of diabetic retinopathy II. Prevalence and risk of diabetic retinopathy when age at diagnosis is less than 30 years. Arch Ophthalmol 1984;102:520-6.

7. D'amicco DJ. Diseases of the retina. N Engl J Med 1994:331:95-106.

8. The Diabetes Control and Complications Trial / Epidemiology of Diabetes Interventions and Complications Research Group. Retinopathy and nephropathy in patients with type 1 diabetes four years after a trial of intensive therapy. N Engl J Med 2000;342:381-9.

9. US Renal Data System. USRDS 2003 Annual Data Report: Atlas of End-Stage Renal Disease in the United States. Bethesda: National Institute of Diabetes and Digestive and Kidney Diseases; 2003.

10. Finnish Registry for Kidney Diseases. Report 2004. Helsink, Finland: Finnish Registry for Kidney Diseases; 2005. Available at: <http://www.musili.fi/smtr/english>. Accessed March, 2006.

11. Parving HH, Oxenboll B, Svendsen PA, Christiansen JS, Andersen AR. Early detection of patients at risk of developing diabetic nephropathy: A longitudinal study of urinary albumin excretion. Acta Endocrinol 1982;100:550-5.

12. Viberti GC, Hill RD, Jarrett RJ, Argyropoulos A, Mahmud U, Keen $\mathrm{H}$. Microalbuminuria as a predictor of clinical nephropathy in insulin-dependent diabetes mellitus. Lancet 1982:1:1430-2.

13. Mogensen $\mathrm{CE}$, Christensen CK. Predicting diabetic nephropathy in insulin-dependent patients. N Engl J Med 1984;311:89-93.

14. Hovind P, Tarnow L, Rossing P, Jensen BR, Graae M, Torp I, et al. Predictors of the development of microalbuminuria and macroalbuminuria in patients with type 1 diabetes: Inception Cohort Study. BMJ 2004:328:1105-8.

15. Dahlquist G, Stattin E-L, Rudberg S. Urinary albumin excretion rate and glomerular filtration rate in the prediction of diabetic nephropathy: a long-term follow-up study of childhood onset type 1 diabetic patients. Nephrol Dial Transplant 2001; 16:1382-6.

16. Arun CS, Stoddart J, Mackin P, Macleod JM, New JP, Marshall SM. Significance of microalbuminuria in long-duration type 1 diabetes. Diabetes Care 2003;26:2144-9.

17. Finne P, Reunanen A, Stenman S, Groop PH, GrönhagenRiska C. Incidence of end-stage renal disease in patients with type 1 diabetes. JAMA 2005;294:1782-7.

18. National Institutes of Health, National Heart, Lung, and Blood Institute. Clinical guidelines on the identifications, evaluation, and treatment of overweight and obesity in adults: The Evidence Report. Obes Res 1998;6:51S-209.
19. American Diabetes Association. Standards of medical care in diabetes-2006. Diabetes Care 2006;29:S4-42.

20. Friedewald WT, Levy RI, Fredrickson DS. Estimation of the concentration of low-density lipoprotein cholesterol in plasma, without use of the preparative ultracentrifuge. Clin Chem 1972;14:499-502.

21. Early Treatment Diabetic Retinopathy Study Research Group. Early treatment diabetic retinopathy study design and baseline patient characteristics. ETDRS report number 7. Ophthalmology 1991;98:741-56.

22. Schwartz GJ, Gauthier B. A simple estimate of glomerular filtration rate in adolescent boys. J Pediatr 1985;106:522-6.

23. Cockcroft DW, Gault HM. Prediction of creatinine clearence from serum creatinine. Nephron 1976;16:31-41.

24. Porta M, Sjoelie A-K, Chaturvedi N, Stevens L, Rottiers R, Veglio $M$, et al. Risk factors for progression to proliferative diabetic retinopathy in the EURODIAB Prospective Complications Study. Diabetologia 2001;44:2203-9.

25. The Diabetes Control and Complications Trial Research Group. The effect of intensive treatment of diabetes on the development and progression of long-term complications in insulin-dependent diabetes mellitus. N Engl J Med 1993:329:997-86.

26. Krolewski AS, Canessa M, Warran JH, Laffel LMB, Christlieb $A R$, Knowler WC, et al. Predisposition to hypertension and susceptibility to renal disease in insulin-dependent diabetes mellitus. N Engl J Med 1988;318:140-5.

27. Mogensen CE, Schmitz A, Christensen CK. Comparative renal pathophysiology relevant to IDDM and NIDDM patients. Diabetes Metab Rev 1988:4:453-83.

28. Klein R, Klein BEK, Moss SE, Davis MD, DeMets DL. Is blood pressure a predictor of the incidence or progression of diabetic retinopathy? Arch Intern Med 1989;149:2427-32.

29. Norgaard K, Feldt-Rasmussen B, Deckert T. Is hypertension a major independent risk factor for retinopathy in type 1 diabetes? Diabet Med 1991;8:334-7.

30. Grunwald JE, Brucker AJ, Schwartz SS, Braunsteins SN, Baker L, Petrig BL, et al. Diabetic glycemic control and retinal blood flow. Diabetes 1990;39:602-7.

31. Miljanovic B, Glynn RJ, Nathan DM, Manson JE, Schaumberg DA. A prospective study of serum lipids and risk of diabetic macular edema in type 1 diabetes. Diabetes 2004;53:288392.

32. Thomas MC, Rosengard-Bärlund M, Mills V, Rönnback M, Thomas S, Forsblom $C$, et al. Serum lipids and the progression of nephropathy in type 1 diabetes. Diabetes Care 2006;29:317-22.

33. Pennathur S, Ido Y, Heller JI, Byun J, Danda R, Pergola P. Reactive carbonyls and polyunsaturated fatty acids produce a hydroxyl radical-like species: a potential pathway for oxidative damage of retinal proteins in diabetes. J Biol Chem 2005;280:22706-14.

34. Yip JW, Jones SL, Wiseman MJ, Hill C, Viberti G. Glomerular hyperfiltration in the prediction of nephropathy in IDDM: A 10-year follow-up study. Diabetes 1996;45:1729-33.

35. Rossing $\mathrm{P}$, Hougaard $\mathrm{P}$, Parving $\mathrm{H}-\mathrm{H}$. Risk factors for development of incipient and overt diabetic nephropathy in type 1 diabetic patients: A 10-year prospective observational study. Diabetes Care 2002;25:859-64.

36. Chavers BM, Mauer SM, Ramsay RC, Steffes MW. Relationship between retinal and glomerular lesions in IDDM patients. Diabetes 1994;43:441-6.

37. Klein R, Zinman B, Gardiner R, Suissa S, Donnelly S, Sinaiko $A R$, et al. The relationship of diabetic retinopathy to preclinical diabetic glomerulopathy lesions in type 1 diabetic patients: The renin-angiotensin system study. Diabetes 2005;54:527-33.

38. Poulsen PL, Bek T, Ebbehloh E, Hansen KW, Mogensen CE. 24-h ambulatory blood pressure and retinopathy in normoalbuminuric IDDM patients. Diabetologia 1998;41:105-10.

39. Levey AS, Coresh J, Balk E, Kausz AT, Levin A, Steffes MW. National Kidney Foundation guidelines for chronic kidney disease: evaluation, classification, and stratification. Ann Intern Med 2003:139:137-47. 
40. Mogensen CE. Long-term anti-hypertensive treatment inhibiting progression of diabetic nephropathy. BMJ 1982;285:685-8.

41. Parving $\mathrm{HH}$, Andersen AR, Smidt UM, Hommel E, Mathiesen ER, Svendsen PA. Effect of anti-hypertensive treatment on kidney function in diabetic nephropathy. BMJ 1987;294:1443-7.

42. ACE Inhibitors in Diabetic Nephropathy Trialist Group. Should all patients with type 1 diabetes mellitus and microalbuminuria receive angiotensin-converting enzyme inhibitors? A meta-analysis of individual patient data. Ann Intern Med 2001;134:370-9.

43. Diabetic Retinopathy Study Research Group. Photocoagulation treatment of proliferative diabetic retinopathy: clinical application of Diabetic Retinopathy Study (DRS) findings, DRS report number 8. Ophthalmology 1981;88:583-600.

44. Early Treatment Diabetic Retinopathy Study Research Group. Early photocoagulation for diabetic retinopathy: ETDRS report number 9. Ophthalmology 1991;98:766-85.

45. The Diabetic Retinopathy Vitrectomy Study Research Group. Early vitrectomy for severe proliferative diabetic retinopathy in eyes with useful vision: clinical application of results of a randomized trial - Diabetic Retinopathy Vitrectomy Study report 4. Ophthalmology 1988;95:1321-34.

46. The UK Prospective Study Group. Tight blood pressure control and risk of macrovascular and microvascular complications in patients with type 2 diabetes: UKPDS 38. BMJ 1998;317:703-13.
47. Chaturvedi N, Sjolie AK, Stephenson JM, Abrahamian $H$, Keipes M, Castellarin A, et al. Effect of lisinopril on progression of retinopathy in normotensive people with type 1 diabetes. Lancet 1998;351:28-31.

48. Chobanian AV, Bakris GL, Black HR, Cushman WC, Green LA, Izzo JL Jr, et al. The seventh report of the Joint National Committee on Prevention, Detection, Evaluation, and Treatment of High Blood Pressure: the $7^{\text {th }}$ report. JAMA 2003;289:2560-72.

Endereço para correspondência:

Henriqueta Galvanin Guidio de Almeida

Departamento de Clínica Médica

Hospital Universitário de Londrina

Rua Robert Koch 60

86038-350 Londrina, PR

Fax: (43) 3371-2000

E-mail: henriqueta@uel.br 